\title{
Editorial
}

\section{Discrete Dynamics in Transportation System 2014}

\author{
Wuhong Wang, ${ }^{1}$ Geert Wets, ${ }^{2}$ Heiner Bubb, ${ }^{3}$ and Yongjun Shen ${ }^{2}$ \\ ${ }^{1}$ Department of Transportation Engineering, Beijing Institute of Technology, Beijing 100081, China \\ ${ }^{2}$ Transportation Research Institute (IMOB), Hasselt University, 3590 Diepenbeek, Belgium \\ ${ }^{3}$ Institute of Ergonomics, Technical University of Munich, 85747 Munich, Germany
}

Correspondence should be addressed to Wuhong Wang; wangwuhong@bit.edu.cn

Received 14 December 2014; Accepted 14 December 2014

Copyright (C) 2015 Wuhong Wang et al. This is an open access article distributed under the Creative Commons Attribution License, which permits unrestricted use, distribution, and reproduction in any medium, provided the original work is properly cited.

The modern transportation system is a dynamic, diverse, random, and complex system. It exhibits extremely complex behavior derived from several components: the heterogeneous nature of human behavior, the highly nonlinear group dynamics, and the large system dimensions. Advances in mobility are most clearly illustrated by the spread of motorized transport and the development of the transportation industry, including vehicle manufacturing and its associated infrastructure. However, the rapid growth of traffic volume has also resulted in several negative impacts, such as urban stress, traffic crashes, and environmental pollution. In order to achieve an efficient, safe, and green mobility, it is therefore essential to model and analyze the discrete states and the dynamic changes of the modern transportation system.

The focus of this annual special issue is to foster links between basic and applied research relating to discrete dynamics problems in transportation systems. We invited original contributions in both new theoretical developments and studies of practical implementations, which propose and investigate novel dynamic and diverse models to reveal the mechanism of transportation system. Totally, 29 articles are included in this special issue, with topics including intelligent transportation system, dynamic problem in urban transport system, transit and rail systems operation, application of chaos in traffic flow, discrete and stochastic theory of transport system, vehicle active safety and intelligent vehicle, discrete optimization methods in traffic system, traffic operations, management and control, energy, transport policy and economics, driving behavior, and driver assistance system.

F. Feng and Q. Zhang constructed a coevolution equation and an order parameter model to analyze the multimodal transport system, and the method was illustrated by using Chinese multimodal transport system as an example.

$\mathrm{J}$. Xu et al. investigated a route planning model for passengers in subway station during peak hours, and the model was applied to a three-layer transfer underground station of Guangzhou subway.

By defining sharp horizontal curves (SHCs) at expressway and collecting the statistics of crashes from 2008 to 2012 on $2200 \mathrm{~km}$ expressways in Guangdong province, X. Li et al. investigated the spatial distribution of crashes on SHCs and deduced the driving risk distribution function of SHCs at expressway. The results provided a theoretical basis to enhance expressway safety management and to improve driving safety on SHCs.

C. Mao et al. investigated the impacts of distressed pavement on driving safety. By analyzing drivers' characteristics, driving behavior, and braking characteristics of vehicles, the relation between the static legibility distance and the dynamic legibility distance of the distressed pavement was revealed, and the minimum safety legibility distance was studied.

$H$. Chen et al. performed a study to analyze the influencing factors in travel mode choice for migrant workers in Xian, China. The results showed that factors such as age, education level, and monthly gross income had a significant impact on travel choice mode for migrant workers.

H. Jin and S. Li designed a vehicular dynamic stability control system based on the calculation of wheel speed difference of nondrive wheels. Different simulation tests were conducted afterwards.

Using the travel data obtained from a survey in the city of Shangyu, China, M. Yang et al. studied the gender 
role-based differences in activity-travel behavior. Binary and ordered logistic regression models segmented by gender were developed for the evaluation. Some interesting findings were obtained.

H. Qin et al. used information display board to design an experimental survey and dynamic decision process data are retrieved under a multimode choice scenario with car, bus and subway, park, and ride. The authors concluded that the most commonly used decision strategy for travelers is the combined strategy under the multimode choice scenario.

L. Lu et al. examined characteristics of e-bike fatal crashes on urban highways in China, using the three-year crash reports (2010-2012) of Taixing city. Some valuable findings were obtained.

Y. Ren et al. investigated drivers' eye-movement characteristics when driving around both left- and right-hand curves via on-road experiment. The results showed that drivers' gaze direction fluctuates around the reference axis and the fixation points are distributed in the region centered on the horizontal gaze position rather than a particular point that has geographical meanings.

R. Liu et al. proposed an overall track quality assessment method for Beijing Metro and determined the overall track quality standards by means of a statistical method, which have been approved to put into practice in China.

J. Xu et al. performed an experimental study on lateral acceleration of cars in different environments. The distribution of the lateral acceleration of a passenger car was obtained from twelve highways in Sichuan with different design speeds and topographies. The effects of curve radius and driving speed on lateral acceleration were analyzed, respectively.

By determining the most important factors in analyzing pedestrian-vehicle conflict, W. Cheng et al. proposed a pedestrian safety conflict index to evaluate the safety level of crossing pedestrians at signalized intersections. The model was then applied to the evaluation of two intersections in the city of Changchun, China.

F. Shi proposed an idea of stratified sampling, which can be used to effectively calculate the sampling rate for resident travel surveys. The method was applied to the main urban area of Kunshan city in Jiangsu province, China.

L. Cheng and F. Han investigated the optimal road toll design problem from the perspective of sustainable development. A bilevel optimization model was designed with the upper level problem to maximize the sustainable development index and the lower level problem to depict travelers' route choice behavior under a certain road toll scheme. The proposed model was effectively applied to the Nguyen-Dupuis network.

Y. Liu and Y. Yu proposed a distributed dynamic traffic assignment strategy for intelligent traffic management, which not only could consider the traveler's preference, but also could achieve a rapid response to the emergency incident in local area.

H. Dai et al. proposed a traffic congestion pricing model for a dual-mode urban transportation network. The model was conducted by solving a bilevel programming problem, in which the delay and emission of vehicles were considered.
M. Huan and X. Yang applied a reliability analysis to explore the red-light running behavior of bicyclists at urban intersections. The authors concluded that bicyclists' safety crossing reliabilities decrease as their waiting times increase.

Y. Yan et al. investigated the vehicle's stability region based on an improved vehicle nonlinear dynamic model using energy function. The model was verified by testing the vehicle snake shape dynamic behavior.

T. Zhang et al. introduced the China Road Assessment Programme (ChinaRAP) model for crash risk assessment. A pilot application was provided afterwards.

By extracting running trajectories of left-turning vehicles and analyzing distribution characteristics of trajectories, velocity changing characteristics, and flow changing characteristics, F. Wei et al. developed a left-turning vehicle's trajectory model and proposed an idea of setting left-turning guide line at the intersection.

M. Du et al. proposed an improved sensitivity analysis based method to estimate the urban transportation network capacity. The results showed that the proposed method could obtain good suboptimal solutions from different starting points.

X. Li et al. proposed a method of axle box bearing fault diagnosis based on multifeature parameters, which includes weak fault signal extraction, feature extraction, and fault recognition. A series of tests was carried out to verify the proposed method.

F. Feng et al. investigated the fault tolerance of railway networks in China and both the macroscopic optimization model and the microscopic optimization model were proposed and applied.

P. Jiao et al. investigated the residence and workplace location choices in Beijing by using mixed logit models. The authors concluded that a joint choice model which combines the residence location and workplace together fits the data the best.

L. Deng et al. developed a rail transit line fare decision support system by solving an optimization model with different objective functions. Some suggestions were given on the selection of flat fare or distance-based fare for the case of Changsha Metro Line 2.

Y. Zhang et al. investigated the impacts of directional and nondirectional auditory warning information in a collision warning system on driving behavior. The results showed that auditory warnings with directional information should be adopted as they are useful to help drivers to avoid collision events and to cross intersections more safely.

S. Sun et al. addressed the vehicle routing problem in large-scale urban transportation networks with stochastic time-dependent travel times. Numerical experiments were conducted on the urban transportation network of Shenzhen, China.

J. Teng and W. Jin developed a bus operation control system to dynamically adjust bus speed, bus dwell time, and traffic signal timings along the path of a running bus, and a simulation platform was developed to evaluate the proposed control system with the actual data collected from the bus route number 210 in Shanghai. 
This annual special issue has offered the opportunity to stimulate interdisciplinary research, exchange ideas, and interact with many of the experts in the research area about discrete dynamics in transportation system. We believe this special issue will contribute nicely to the development of transportation behaviour, intelligent transportation system, and traffic safety.

Wuhong Wang

Geert Wets

Heiner Bubb

Yongjun Shen 


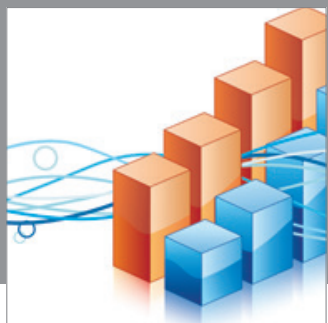

Advances in

Operations Research

mansans

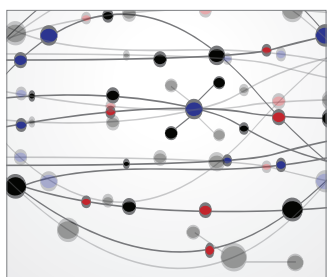

The Scientific World Journal
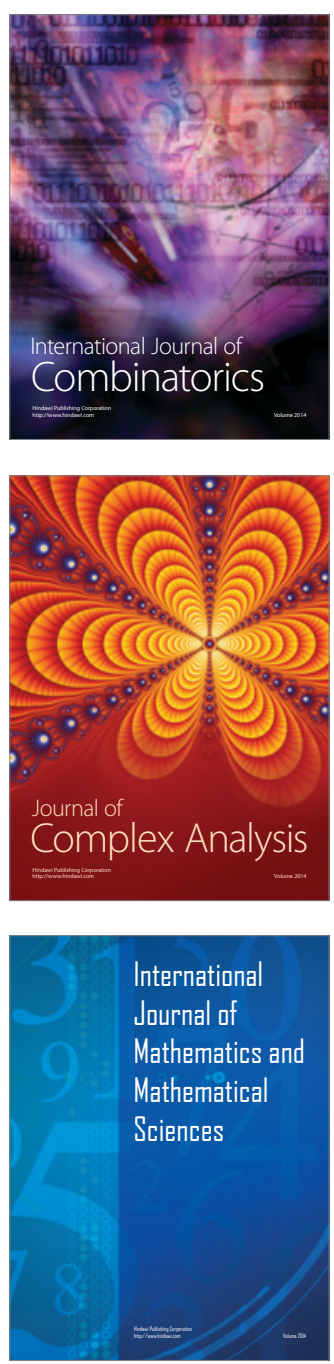
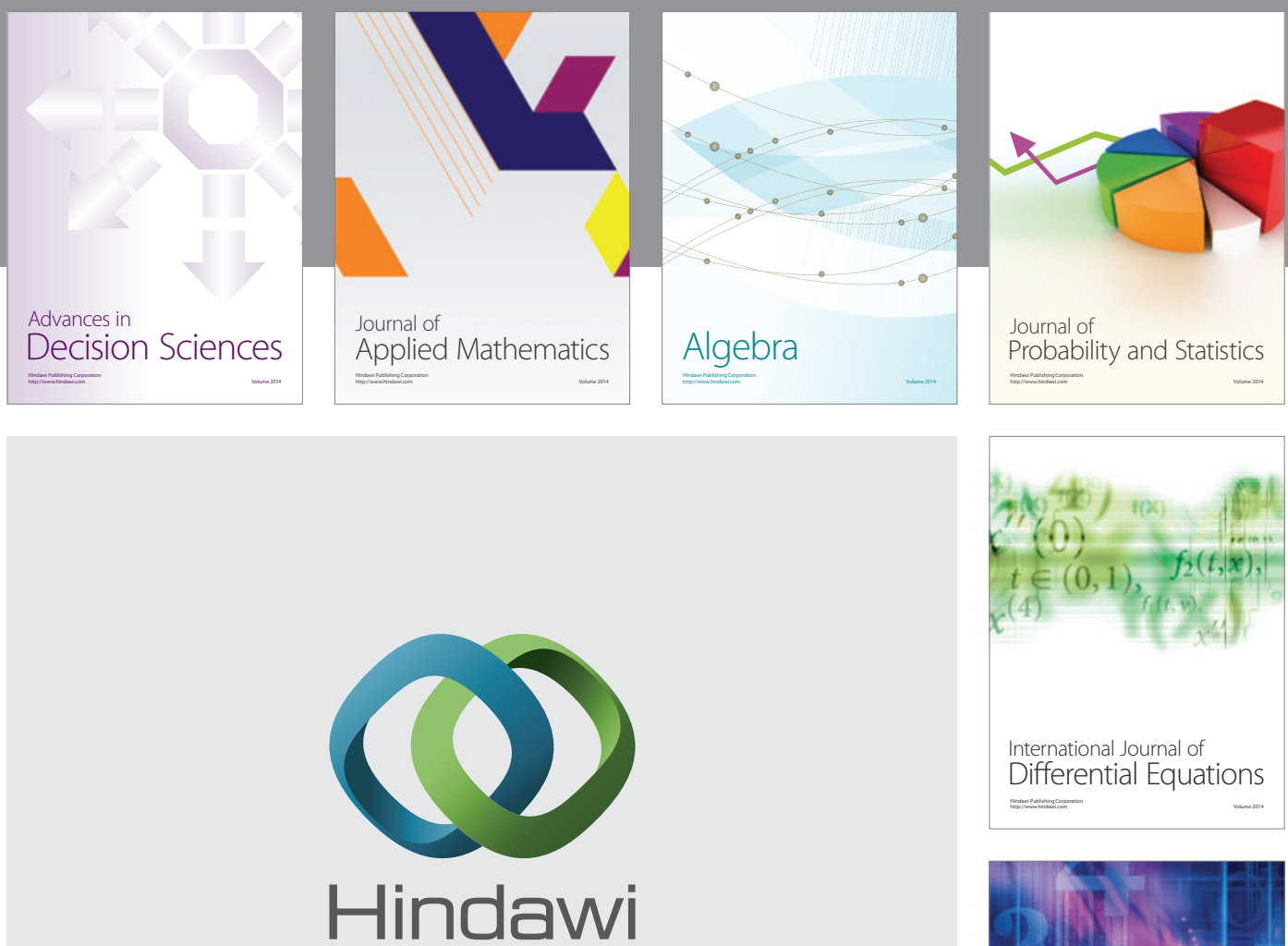

Submit your manuscripts at http://www.hindawi.com
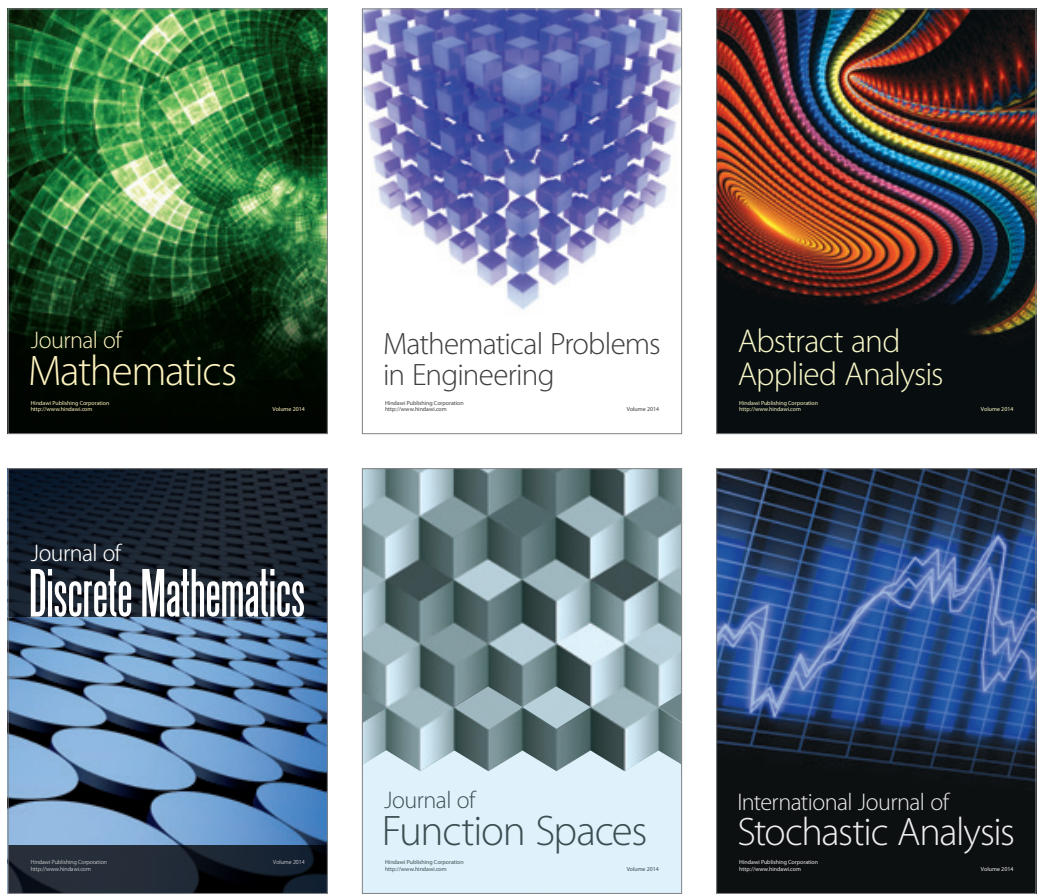

Journal of

Function Spaces

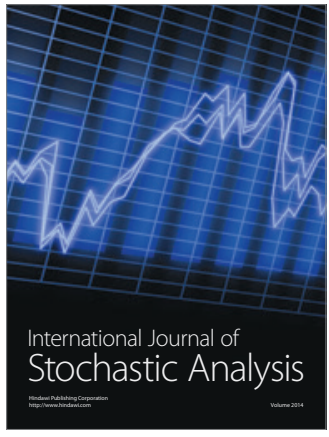

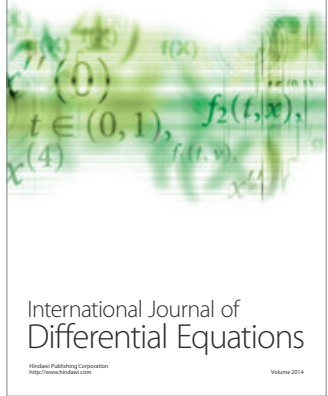
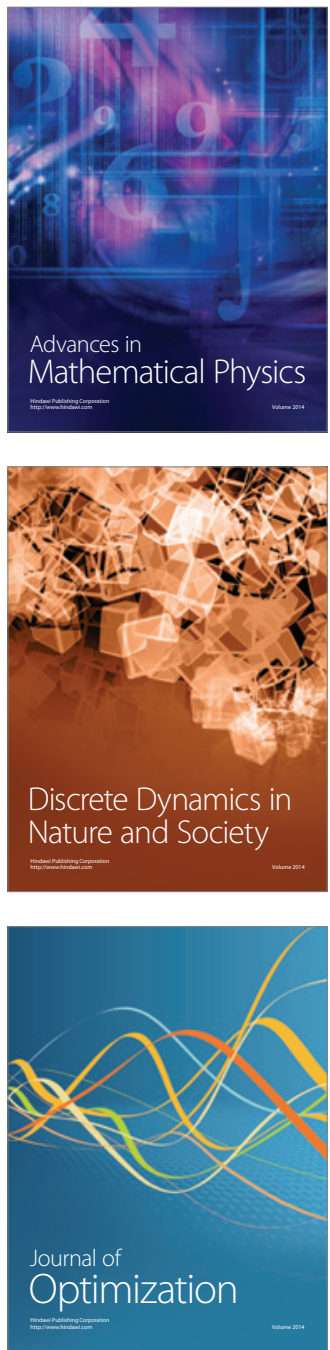